Proceedings of the XVI National Conference on Superconductivity and Strongly Correlated Systems, Zakopane 2013

\title{
Stability of Bilayer Superconductors against Thermomagnetic Avalanche
}

\author{
S.V. VAsiliev ${ }^{a}$, O.M. Chumak ${ }^{a, b}$, V.V. Chabanenko ${ }^{a, c, *}$, F. PÉrez-Rodríguez ${ }^{c}$ \\ AND A. NABIAŁEK ${ }^{d}$
}

${ }^{a}$ O. Galkin Institute of Physics and Technology, National Academy of Sciences,

72 R. Luxemburg, 83114, Donetsk, Ukraine

${ }^{b}$ Donetsk National University, 24 Universitetskaya, 83055, Donetsk, Ukraine

${ }^{c}$ Instituto de Física, Benemérita Universidad Autónoma de Puebla,

Apartado Postal J-48 Puebla, 72570 Puebla, México

${ }^{d}$ Institute of Physics, PAS, al. Lotników 32/46, 02-668 Warsaw, Poland

\begin{abstract}
The problem of stability of bilayer type II superconductor with different critical current densities of inner part and coating was considered. The optimum thickness of surface layer enabling the maximal increase of the field of first flux jump was found. The field of instability for such bilayer structure was calculated for NbTi superconductor. An increase of the field of the first flux jump by about $60 \%$ has been found for optimal coating thickness.
\end{abstract}

DOI: 10.12693/APhysPolA.126.A-84

PACS: 74.25.Op, 74.72.-h, 74.25.Sv

\section{Introduction}

The analysis of causes and patterns of occurrence of low launch fluctuations - flux jumps (from tens to hundreds of thousands magnetic flux quanta), which can grow into a giant magnetothermal avalanche, is very important in the investigation of the stability of critical state. Thermomagnetic instabilities, which lead to a sudden failure of the critical state, are the major factor limiting the high current technical applications of bulk superconductors. Thermomagnetic instabilities in superconductors are identified as giant jumps of properties (resistance, temperature, speed of sound, magnetic flux, magnetization, etc.). They often lead to a melting of some elements of the devices and to irreversible consequences that damage expensive electrical equipment (solenoids, motors, energy storage).

Flux jumping in hard type-II superconductors is now fairly well understood, and several stabilization techniques have been developed for magnet applications. These techniques involve subdividing the superconductor into filaments within a normal metal matrix, and enable magnet conductors to operate at currents close to the critical values of short samples [1]. Diameters of superconductors thread do not exceed $0.1 \mathrm{~mm}$, and usually amount to several $\mu \mathrm{m}$ in such composite superconductors. Flux jumps appear, when the diameter of the thread increases above some critical value $\left(d_{\mathrm{c}}\right)$. This critical diameter is connected with the field of the first flux jump and with the value of critical current density $d_{\mathrm{c}}=\frac{2 B_{1 j}}{\mu_{0} j_{\mathrm{c}}}$, where $B_{1 j}$ - field of the first flux jump, $j_{\mathrm{c}}-$ critical current density and $\mu_{0}=4 \pi \times 10^{-7} \mathrm{H} / \mathrm{m}$.

${ }^{*}$ corresponding author; e-mail: vikchabanenko@gmail.com
However, there is another way to increase the stability of the critical state in hard type-II superconductors, suggested by Suttons in Ref. [2]. He experimentally observed that the flux jump field is increased in a laminated superconductor, in which the surface layer has lower critical current density than the underlayer. From qualitative considerations, criteria of stability have been obtained in this situation.

Recently, in paper [3], there was found an increase of stability of the critical state of the superconducting wire with a core and a surface layer of $\mathrm{Nb}_{3} \mathrm{Sn}$ and $\mathrm{Nb}$, respectively. In present article, we consider this problem quantitatively. We have obtained criteria of stability in such bilayer superconductors.

\section{Model and calculation}

We considered bilayer superconductors consisting of inner part with critical current density $j_{2}(T)$ (SC-II), and of thin surface layer of thickness $\delta_{1}$ with lower value of critical current density $j_{1}(T)$ (SC-I). We investigated the stability of such composite by methods proposed by Mints and Rakhmanov in their review [4]. For the critical state of type-II superconductor, time dependent distributions of magnetic and electric field as well as of the temperature can be described by the following system of partial differential equations:

$$
\left\{\begin{array}{l}
\operatorname{rot} \boldsymbol{B}=\mu_{0} \boldsymbol{j}=\mu_{0} \frac{\boldsymbol{j}_{\mathrm{c}}(T) \cdot \boldsymbol{E}}{|\boldsymbol{E}|}+\mu_{0} \sigma_{f} \boldsymbol{E}, \\
\operatorname{rot} \boldsymbol{E}=-\frac{\partial \boldsymbol{B}}{\partial t} \\
C(T) \frac{\partial T}{\partial t}=\kappa \Delta T+\boldsymbol{j}(T) \cdot \boldsymbol{E} \\
\boldsymbol{j}=j_{\mathrm{c}}(T) \frac{\boldsymbol{E}}{|\boldsymbol{E}|}+\sigma_{f} \boldsymbol{E}
\end{array}\right.
$$

where $T$ - temperature, $\boldsymbol{B}-$ magnetic induction, $\boldsymbol{E}$ - electric field, $j_{\mathrm{c}}$ - critical current density, $C(T)$ specific heat per unit volume, $\kappa$ - thermal conductivity and $\sigma_{f}$ - flux-flow electrical conductivity. 
Let us introduce the parameter $\tau$ equal to the ratio between the time of magnetic diffusion $\left(t_{\mathrm{m}}\right)$ and the time of thermal diffusion $\left(t_{\mathrm{t}}\right): \tau=\frac{D_{\mathrm{t}}}{D_{\mathrm{m}}}=\frac{\mu_{0} \sigma_{f} \kappa}{C(T)}, D_{\mathrm{t}}$ - thermal diffusivity, $D_{\mathrm{m}}$ - magnetic diffusivity. For most of type II superconductors (especially conventional), the magnetic diffusivity $D_{\mathrm{m}}$ is very much greater than the thermal diffusivity $D_{\mathrm{t}}$, and then the parameter $\tau \ll 1$. This situation is usually called "locally adiabatic" or $\tau$-zero approach $(\tau=0)$. The opposite so-called "dynamic" limit $\tau \gg 1$ is realized in composite superconductors. As shown in Ref. [4] in $\tau$-zero approach, system (1) can be simplified, and written in next form

$$
\left\{\begin{array}{l}
\operatorname{rot} \boldsymbol{B}=\mu_{0} \frac{\boldsymbol{j}_{\mathrm{c}}(T) \cdot \boldsymbol{E}}{|\boldsymbol{E}|}, \\
\operatorname{rot} \boldsymbol{E}=-\frac{\partial \boldsymbol{B}}{\partial t}, \\
C(T) \frac{\partial T}{\partial t}=\left(\frac{\boldsymbol{j}_{\mathrm{c}}(T) \cdot \boldsymbol{E}}{|\boldsymbol{E}|}, \boldsymbol{E}\right) .
\end{array}\right.
$$

We assume that our superconductor (with $j_{\mathrm{c}}=j_{2}(T)$ ) is covered by modified layer of thickness $\delta_{1}$ (with $j_{\mathrm{c}}=$ $\left.j_{1}(T)\right)$, and occupies half-infinite space. We introduce the critical currents' ratio $K=j_{2} / j_{1}$. The external magnetic field is directed along the surface of such superconducting structure. In this situation, system (2) reduces to one-dimensional problem. Schematic distribution of magnetic field is shown in Fig. 1. In this case, system (2) can be written as follows:

$$
\left\{\begin{array}{l}
\frac{\partial B}{\partial x}=-\mu_{0} j_{\mathrm{c}}(x), \\
\frac{\partial E}{\partial x}=-\frac{\partial B}{\partial t}, \\
C(T) \frac{\partial T}{\partial t}=j_{\mathrm{c}}(x) E, \\
j_{\mathrm{c}}(x)= \begin{cases}j_{2}(T) / K, & 0 \leq x \leq \delta_{1}, \\
j_{2}(T), & \delta_{1}<x \leq \delta_{2} .\end{cases}
\end{array}\right.
$$

We investigated the stability of this system against the small perturbations of temperature, magnetic and electric field. With this purposes, we represented $B, E$, and $T$ in the next form

$$
\left\{\begin{array}{l}
B(x, t)=B_{0}(x)+b(x, t), \\
E(x, t)=E_{0}(x)+\varepsilon(x, t), \\
T(x, t)=T_{0}+\theta(x, t),
\end{array}\right.
$$

where $B_{0}(x), E_{0}(x)$ - stationary distribution of magnetic and electric field, $T_{0}$ - initial temperature of superconductor (usually equal to the temperature of coolant bath), and $b(x, t), \varepsilon(x, t), \theta(x, t)$ - infinitesimal perturbations. If the critical current densities do not depend on magnetic field,

$$
B_{0}(x)=\left\{\begin{array}{l}
B_{\alpha}-\frac{\mu_{0} j_{2}(T)}{K} x, 0 \leq x \leq \delta_{1}, \\
\left(B_{\alpha}-\frac{\mu_{0} j_{2}(T)}{K} \delta_{1}\right)-\mu_{0} j_{2}(T)\left(x-\delta_{1}\right), \\
\delta_{1}<x \leq \delta_{2}, \\
0, x>\delta_{2}, \\
\delta_{2}=\frac{B_{\alpha}-\mu_{0} \delta_{1}\left(\frac{1}{K}-1\right) j_{2}(T)}{\mu_{0} j_{2}(T)},
\end{array}\right.
$$

where $B_{\alpha}$ - the external magnetic field on the surface of bilayer superconductor. This distribution of magnetic field is shown in Fig. 1.

After linearization of this system, in the case of small perturbations, one obtains

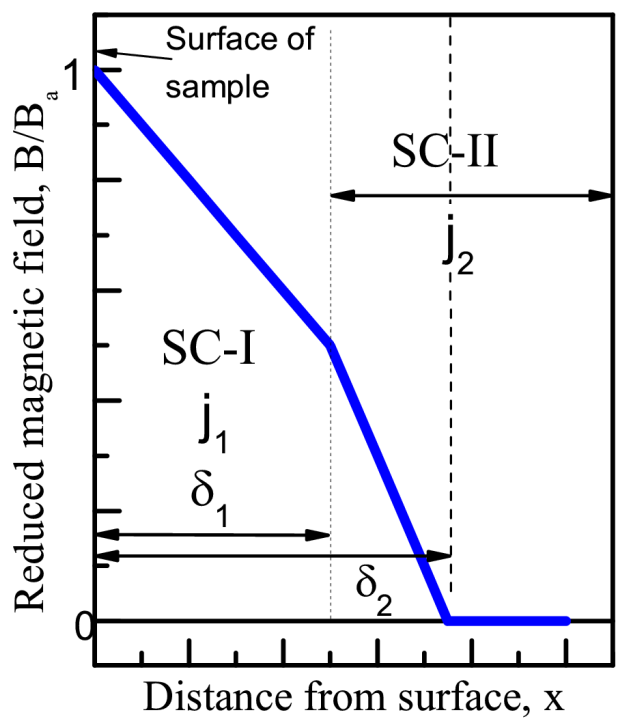

Fig. 1. Schematic distribution of magnetic field in bilayer superconductor with two different critical current densities $\left(j_{1}, j_{2}\right)$ and thicknesses $\left(\delta_{1}, \delta_{2}\right)$.

$$
\left\{\begin{array}{l}
\frac{\partial b}{\partial x}=\mu_{0} Q(x) \theta, \\
\frac{\partial \varepsilon}{\partial x}=-\frac{\partial b}{\partial t}, \\
C\left(T_{0}\right) \frac{\partial \theta}{\partial t}=j_{\mathrm{c}}\left(x, T_{0}\right) \varepsilon, \\
Q(x)=\left|\frac{\partial j_{\mathrm{c}}(x, T)}{\partial T}\right|_{T=T_{0}} \mid .
\end{array}\right.
$$

When instability of the critical state appears, small perturbations of values $b, \varepsilon$ and $\theta$ begin to exponentially increase in time $-b, \varepsilon, \theta \sim \mathrm{e}^{\lambda t}$ and $\operatorname{Re}(\lambda)>0$. Assuming exponential temporal dependence of the fluctuations, one can obtain the following Sturm-Liouville problem:

$$
\left\{\begin{array}{l}
\tilde{\varepsilon}^{\prime \prime}=-f^{2}(x) \tilde{\varepsilon}, \\
\tilde{\varepsilon}^{\prime}(0)=\tilde{\varepsilon}\left(\delta_{2}\right)=0, \\
\varepsilon(x, t)=\tilde{\varepsilon}(x) \mathrm{e}^{\lambda t},
\end{array}\right.
$$

where

$$
f^{2}(x)=\left\{\begin{array}{l}
\frac{\mu_{0}}{C\left(T_{0}\right) K^{2}} j_{2}\left(T_{0}\right)\left|\frac{\partial j_{2}}{\partial T}\right|_{T_{0}} \mid, 0 \leq x \leq \delta_{1}, \\
\frac{\mu_{0}}{C\left(T_{0}\right)} j_{2}\left(T_{0}\right)\left|\frac{\partial j_{2}}{\partial T}\right|_{T_{0}} \mid, \delta_{1}<x \leq \delta_{2} .
\end{array}\right.
$$

This problem can be solved in each region $\left(\left[0, \delta_{1}\right]\right.$ and $\left.\left(\delta_{1}, \delta_{2}\right]\right)$, separately. Using the continuity conditions, the field of the first flux jump can be obtained

$$
\left\{\begin{array}{l}
B_{1 j}\left(T_{0}\right)=B_{0 j}\left(T_{0}\right) \\
\times\left[1-\frac{2}{\pi} \arctan \left(\frac{\tan \left(\frac{\left.2 \delta_{1}\left|\frac{\partial j_{2}}{\partial T}\right|_{T=T_{0}}\right|^{\pi K C\left(T_{0}\right)} B_{0 j}\left(T_{0}\right)}{K}\right)}{K}\right)\right] \\
\quad+\frac{\mu_{0} j_{2}\left(T_{0}\right) \delta_{1}}{K}, \\
B_{0 j}\left(T_{0}\right)=\frac{\pi}{2} \sqrt{\frac{\mu_{0} C\left(T_{0}\right) j_{2}\left(T_{0}\right)}{\left|\frac{\partial j_{2}}{\partial T}\right|_{T=T_{0}} \mid}}
\end{array}\right.
$$

Assuming linear temperature dependence of the critical current density $j_{2}(T)=j_{02}\left(1-T / T_{\mathrm{c}}\right)\left(T_{\mathrm{c}}-\right.$ critical temperature), it can be found that 


$$
\left\{\begin{array}{l}
B_{1 j}\left(T_{0}\right)=\frac{\mu_{0} j_{02}\left(1-T_{0} / T_{\mathrm{c}}\right) \delta_{1}}{K} \\
+B_{0 j}\left(T_{0}\right)\left[1-\frac{2}{\pi} \arctan \left(\frac{\tan \left(\frac{2 \delta_{1} j_{02}}{\pi K C\left(T_{0}\right) T_{\mathrm{c}}} B_{0 j}\left(T_{0}\right)\right)}{K}\right)\right] \\
B_{0 j}\left(T_{0}\right)=\frac{\pi}{2} \sqrt{\mu_{0} C\left(T_{0}\right)\left(T_{\mathrm{c}}-T_{0}\right)},
\end{array}\right.
$$

where $B_{0 \mathrm{j}}(T)$ - well known expression for field of first jump in conventional case (without coating) [4].

\section{The critical state stability analysis of NbTi bilayer}

We used criterion (9) for NbTi superconductor with next parameters [1]:

$$
\begin{aligned}
C(T) & =288.54 T^{3} \frac{J}{\mathrm{~K} \mathrm{~m}^{3}}, \\
j_{02} & =6 \times 10^{9} \frac{\mathrm{A}}{\mathrm{m}^{2}}, T_{\mathrm{c}} \approx 9.5 \mathrm{~K} .
\end{aligned}
$$

With fixed parameters $T$ and $K$, the field of the first flux
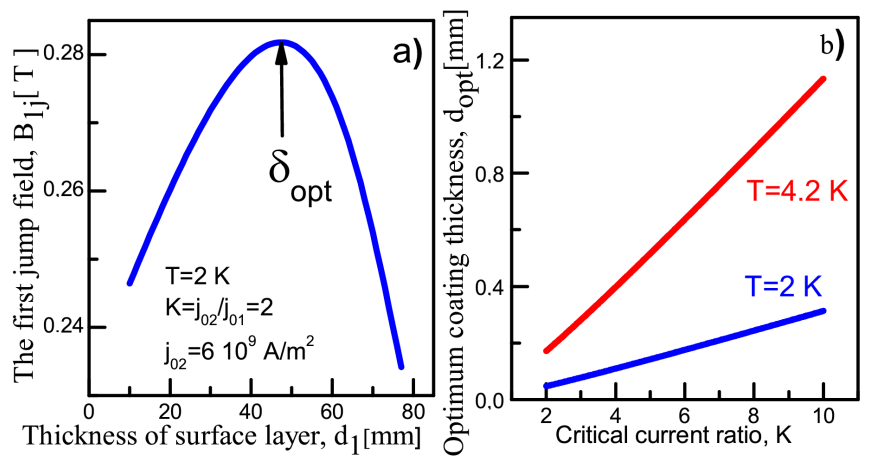

Fig. 2. (a) Dependence of the field of first jump versus thickness of surface layer; (b) dependences of optimal thickness of coating $\left(\delta_{\mathrm{opt}}\right)$ versus critical currents' ratio $(K)$ for different temperatures, $T=2 \mathrm{~K}$ and $T=4.2 \mathrm{~K}$.

jump (9) has non-monotonic dependence on the thickness of surface layer (Fig. 2a). We can find an optimal thickness of the surface layer $\left(\delta_{\text {opt }}<d_{\mathrm{c}}\right)$, which leads to the maximal value of the field of first jump. This value depends on both $T$ and $K$ :

$$
\begin{aligned}
& \delta_{\text {opt }}\left(T_{0}, K\right)=\frac{2}{\pi} \frac{B_{0 j}\left(T_{0}\right)}{\mu_{0} j_{2}\left(T_{0}\right)} K \arccos \frac{1}{\sqrt{1+K}}= \\
& \frac{2}{\pi} d_{\mathrm{c}} \arccos \frac{1}{\sqrt{1+K}} .
\end{aligned}
$$

The dependence of $\delta_{\text {opt }}$ versus $K$ for different temperatures, $T=2 \mathrm{~K}$ and $T=4.2 \mathrm{~K}$, is shown in Fig. $2 \mathrm{~b}$. For $K \gg 1$ this dependence is practically linear.

Temperature dependence of the field of first jump for different optimal thicknesses of the surface layer is shown in Fig. 3 (blue and red line). For comparison, similar dependence in conventional case without coating (black line) is shown.

The influence of the coating on the increase of the critical state stability can be clearly seen in Fig. 4, where we present relative changes of the field of first jump, $\Delta B(T)=\frac{B_{1 j}(T)-B_{0 j}(T)}{B_{0 j}(T)} 100 \%$, for optimized thickness of

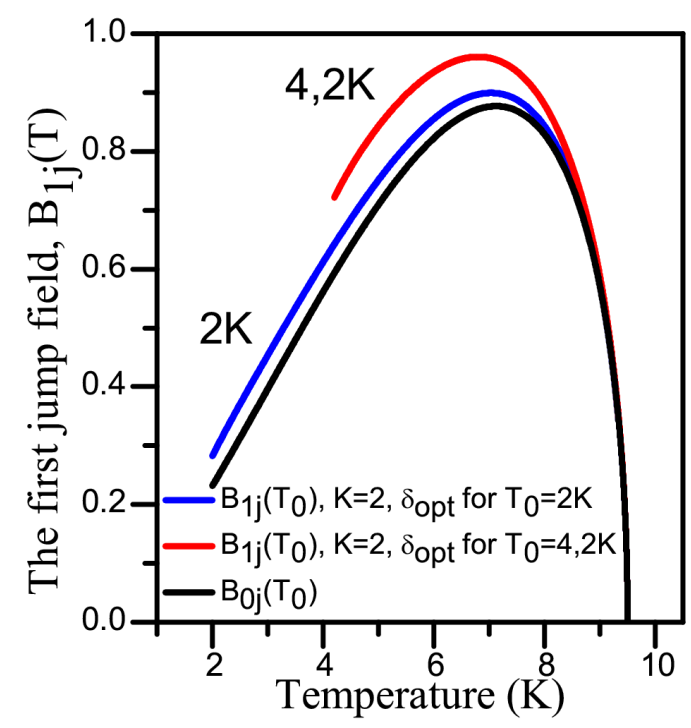

Fig. 3. Temperature dependences of field of first flux jump for different thicknesses of coating. Red line optimum thickness for $T=4.2 \mathrm{~K}$, blue line - for $T=$ $2 \mathrm{~K}$. Black line - field of first flux jump for uncoated superconductor (conventional case).

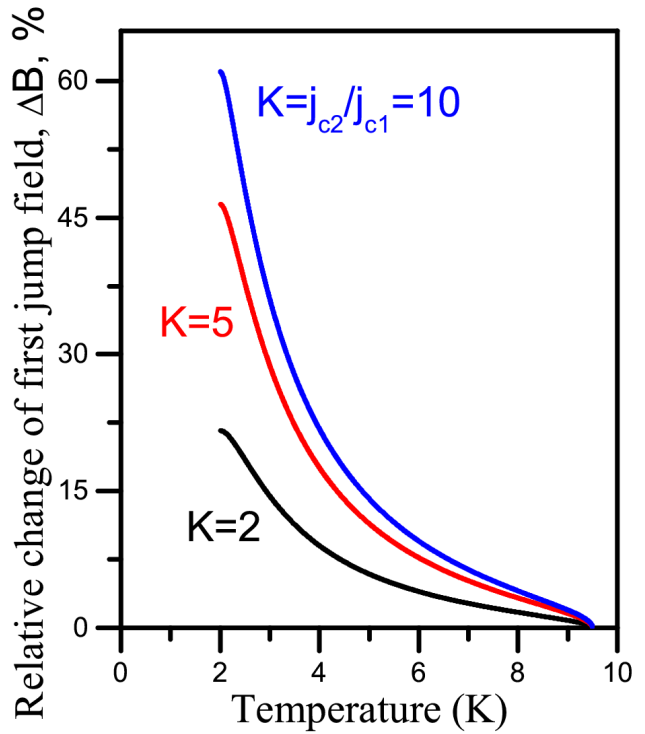

Fig. 4. Relative changes of the field of first jump $(\Delta B)$ versus temperature for different ratios of the critical current densities $\left(K=j_{02} / j_{01}\right)$. These curves are calculated for optimal thickness of surface layer.

surface layer and different ratios of critical current densities.

\section{Conclusion}

Criterion of stability of the critical state for bilayer superconductor has been obtained. This criterion has strong dependence on thickness of coating and on the 
ratio of the critical current densities. Using the coating with lower critical current density, one can increase the field of the first flux jump as well as the critical dimensions of the superconducting elements. In the case of conventional NbTi superconductor for optimal coating, the field of the first flux jump can be increased by about 60 percent.

\section{Acknowledgments}

This work was partially supported by SEP-CONACYT (Mexico) under grant CB-2012-01-183673.

\section{References}

[1] E.W. Collings, Applied Superconductivity, Metallurgy, and Physics of Titanium Alloys, Plenum Press, New York 1986

[2] J. Sutton, J. Appl. Phys. 44, 465 (1973).

[3] P. Fabbricatore, M. Greco, C. Ferdeghini, C. Bernini, U. Gambardella, G. Celentano, A. Devred, Supercond. Sci. Technol. 20, 34 (2007).

[4] R.G. Mints, A.L. Rakhmanov, Rev. Mod. Phys. 53, 551 (1981). 